\title{
High-quality inkjet-printed multilevel interconnects and inductive components on plastic for ultra-low-cost RFID applications.
}

Steven Molesa, David R. Redinger, Daniel C. Huang, and Vivek Subramanian.

Department of Electrical Engineering, University of California Berkeley,

Berkeley, CA 94720-1770, U.S.A.

\section{ABSTRACT}

In recent years, there has been tremendous interest in all-printed electronics as a means of achieving ultra-low-cost electronic circuits with uses in displays and disposable electronics applications such as RFID tags. While there have been a few demonstrations of printed organic transistors to date, there has been little work on the associated passive component and interconnection technologies required to enable the development of all-printed RFID circuits. In particular, low-resistance conductors are crucial to achieve the high-Q inductors necessary for RFID. Here, we demonstrate inkjetted nanoparticle-Au conductors on plastic with sheet resistances as low as $0.03 \mathrm{ohms} / \mathrm{square}$. We describe the optimization of the jetting parameters, and their impact on final film morphology and electrical properties. We also demonstrate a bridging technology based on an inkjetted polyimide interlevel dielectric. Using this process, we demonstrate multilevel interconnect and passive component structures including conductor patterns, crossover bridges, and tapped planar spiral inductors. Together, these represent an important step towards the realization of all-printed RFID.

\section{INTRODUCTION}

Recently, there has been growing interest in the development of printed organic electronics technologies, which are expected to see use in low-cost, flexible displays and disposable electronics applications. Low-cost RFID tags are considered to be a compelling application, since they may be used to replace UPC barcodes on consumer products, ushering in an era of enhanced consumer convenience and warehousing efficiency, through a realization of real-time price and product controls, automated inventory processes, and automated checkout.

All-printed circuit technologies are attractive for several reasons. They eliminate the need for expensive lithography, and also eliminate the need for high-vacuum processing, including PVD, CVD, plasma etching, etc., all of which have major impacts on system cost. Additionally, they use an additive fabrication process, which reduces the waste abatement costs. Thus, they are expected to result in a substantially reduced integrated cost making them suitable for use in disposable consumer products.

In recent years, there have been some demonstrations of printed transistors in plasticcompatible technologies [1]. However, to date, there have been no significant demonstrations of the requisite all-printed passive component technologies. In particular, for RFID, this is a crucial shortcoming, since the requirements imposed on the passive components are tremendous; high-Q inductors and well-behaved capacitors are required for power-coupling and communication [2]. Last year, we demonstrated a plastic-compatible gold nanoparticle-based conductor technology that enabled the printing of low-resistance conductors on plastic for the first time [3]. Here, we optimize and apply this and other materials to inkjet printing applications, and demonstrate a robust multilevel interconnect technology for RFID circuits, and also demonstrate the associated inductive components required for the same. 


\section{EXPERIMENTAL DETAILS}

All experiments were performed using a custom inkjet system, shown in figure 1. The overall testbed consists of translation stages, inkjet dispensers, a hot chuck for heating and cooling the substrate, and software to control the various systems. For all experiments, we used piezoelectric heads manufactured by Microfab, Inc., with nozzle diameters varying from $30 \mu \mathrm{m}$ to $60 \mu \mathrm{m}$. Custom software was used to provide overlay, translation, and head control.

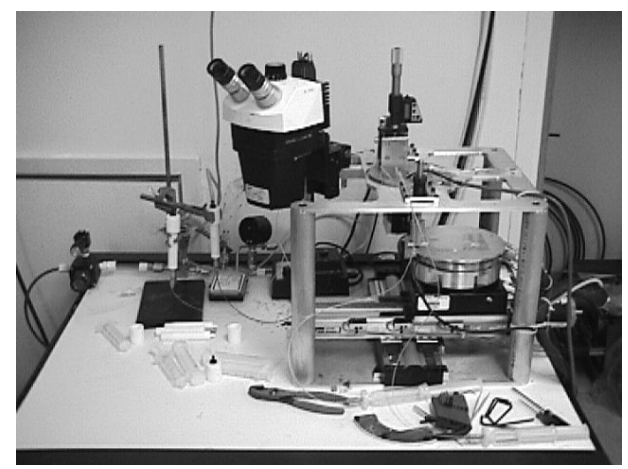

Figure 1: The custom inkjet system for printed electronics fabrication.

To develop the processes for forming inductive components and multilevel interconnects, we performed experiments using metallic nanoparticles [3] for conductor formation, and a commercial polyimide for dielectric formation. We varied the droplet jetting waveform parameters, droplet spacing, choice of solvent, and substrate temperature during printing. Resultant film morphology (as measured using optical micrography, profilometry, and AFM) and electrical conductivity were correlated to these parameters and used to drive the optimization of the processes. These were used to demonstrate inductors and multilevel interconnects.

\section{RESULTS AND DISCUSSION}

The piezo-head waveform parameters were optimized to maximize jetting velocity while ensuring good drop-to-drop stability and the absence of satellite droplets. By standardizing all experimental runs to this baseline, it was possible to specifically examine the impact of various process and materials parameters on film quality, for both the conductor and dielectric films.

\section{Conductor Film Development}

$10 \mathrm{wt} \%$ hexanethiol-encapsulated $1.5 \mathrm{~nm}$ gold nanoparticles were dissolved in toluene. This was inkjet printed onto polyester substrates at room temperature. Due to the velocity of inkjetprinted droplets, a "splash" effect resulted upon impact of the droplet. After the evolution of the splash wave, the evaporation of the toluene resulted in the formation of a coffee-ring or donut structure. This is a known problem with inkjet printing [4]. For conductor development, this is a crucial issue, since it results in the production of rough, films with high sheet resistance. Owing to their roughness, these films are generally unsuitable for use in multilayer interconnect structures, since overlying dielectrics are prone to pin-holes due to the poor coverage of the numerous ridges and valleys. The structure of a typical film showing the coffee-ring effect is presented in figure 2 . 

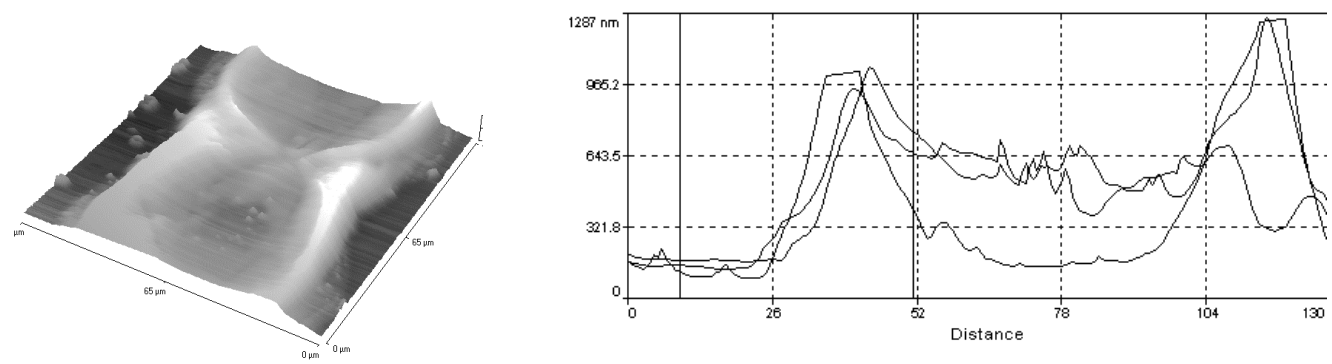

Figure 2: (left) atomic force micrograph showing the characteristics "coffee-ring" structure that results from splashing during droplet deposition, and (right) profilometry of a typical film formed using this process, showing the substantial roughness.

The solution to this problem is to increase the evaporation rate of the solvent upon droplet deposition on the surface, preventing the splash wave from traveling, and hence eliminating the formation of the coffee-ring. This is achieved using substrate heating to enhance the evaporation rate of the solvent at the droplet surface. Indeed, upon raising the substrate temperature to $130^{\circ} \mathrm{C}$, the toluene solvent evaporates almost instantaneously upon droplet deposition, eliminating more than $80 \%$ of the coffee-ring. This is apparent in figure 3.
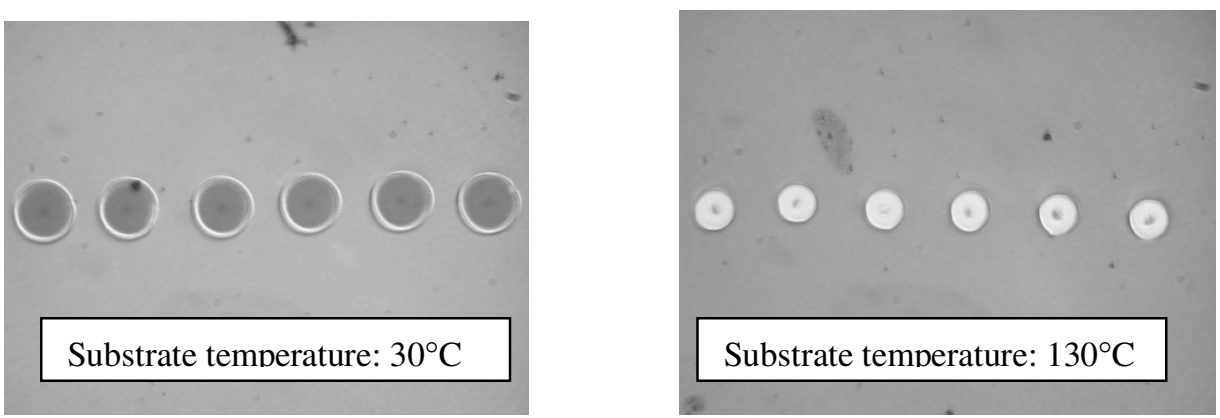

Figure 3: Effect of substrate temperature during deposition on coffee-ring splash effect. Notice the dramatic reduction in the central coffee ring hole in the droplets on the right.

By overlaying droplets with a spacing substantially less than the droplet diameter, it is possible to use successive droplets to "fill" the coffee-ring produced by previous droplets. This results in the formation of films with improved smoothness, and almost complete elimination of the ridges at the edges of the film. Due to the small coffee-ring contribution from each drop, however, the center of a printed line is somewhat thinner than the edges, as shown in figure 4.
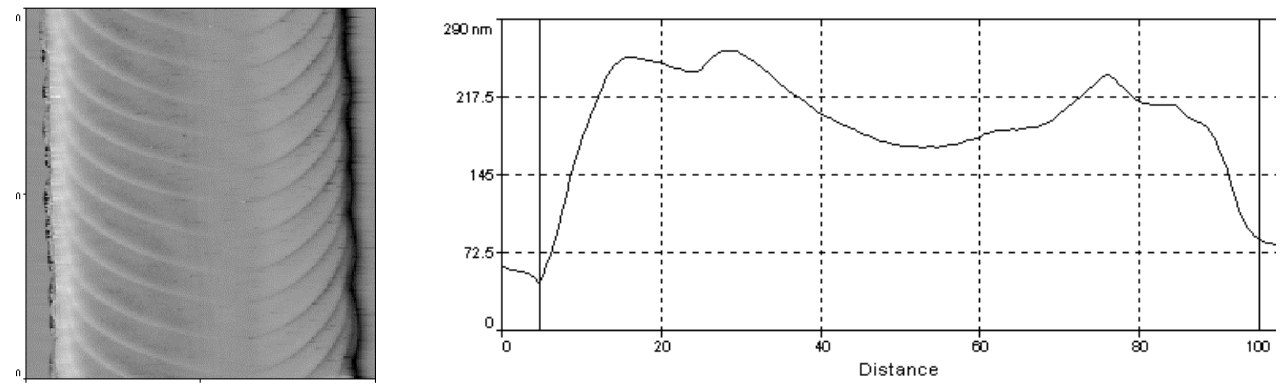

Figure 4: Overlaying of successive drops to reduce the coffee-ring splash effect. 
There is a disadvantage to using substrate heating to reduce the coffee-ring effect, however. To achieve good control on droplet placement, typical inkjet systems maintain a head-tosubstrate distance of less than $2 \mathrm{~mm}$. This results in substantial convective heat transfer to the head, which in turn results in enhanced evaporation of solvent at the nozzle tip. Consequently, there is an increased likelihood of partial and complete clogging of the head, resulting in tremendous process stability and reliability concerns while inkjetting. To solve this problem, we have experimented with a variety of lower-evaporation rate solvents. In particular, we have had excellent success using alpha-terpineol. The use of this solvent has several advantages. First, due to the slower evaporation rate of the solvent at the nozzle, it offers excellent clog resistance. Second, due to its higher viscosity compared to toluene, it provides a larger optimization window for substrate-heating-based control of the coffee-ring, and enables complete elimination of the coffee-ring effect at $160^{\circ} \mathrm{C}$. By syncopating droplets, it is possible to produce extremely smooth lines, with no ridges and negligible cross-sectional thickness variation, as shown in figure 5.
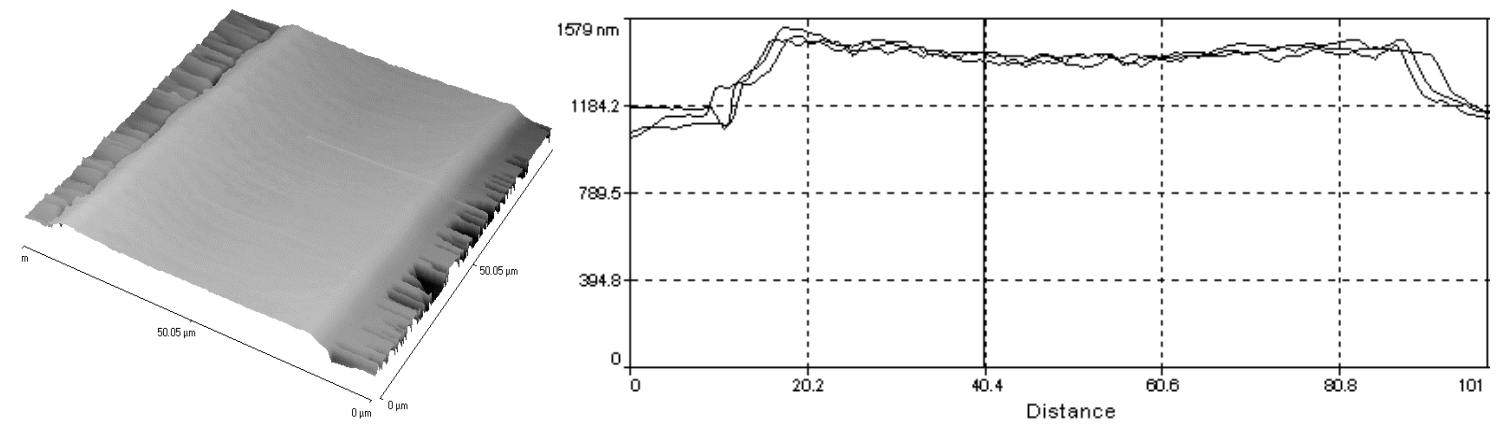

Figure 5: Smooth conductor lines obtained by proper optimization of temperature and solvent.

Printing at elevated temperatures using alpha-terpineol has an additional advantage. Due to the higher-temperatures, the alkanethiol is removed more efficiently, resulting in lower sheet resistance, as shown in figure 6 . This removal of the alkanethiol has been previously identified as an important requirement for producing low-resistance films [3]. Indeed, by further optimization, conductivities as high as $70 \%$ of bulk gold have been obtained in thinner films. Sheet resistances as low as $23 \mathrm{~m} \Omega$ /square have been obtained in $1 \mu \mathrm{m}$ thick films.

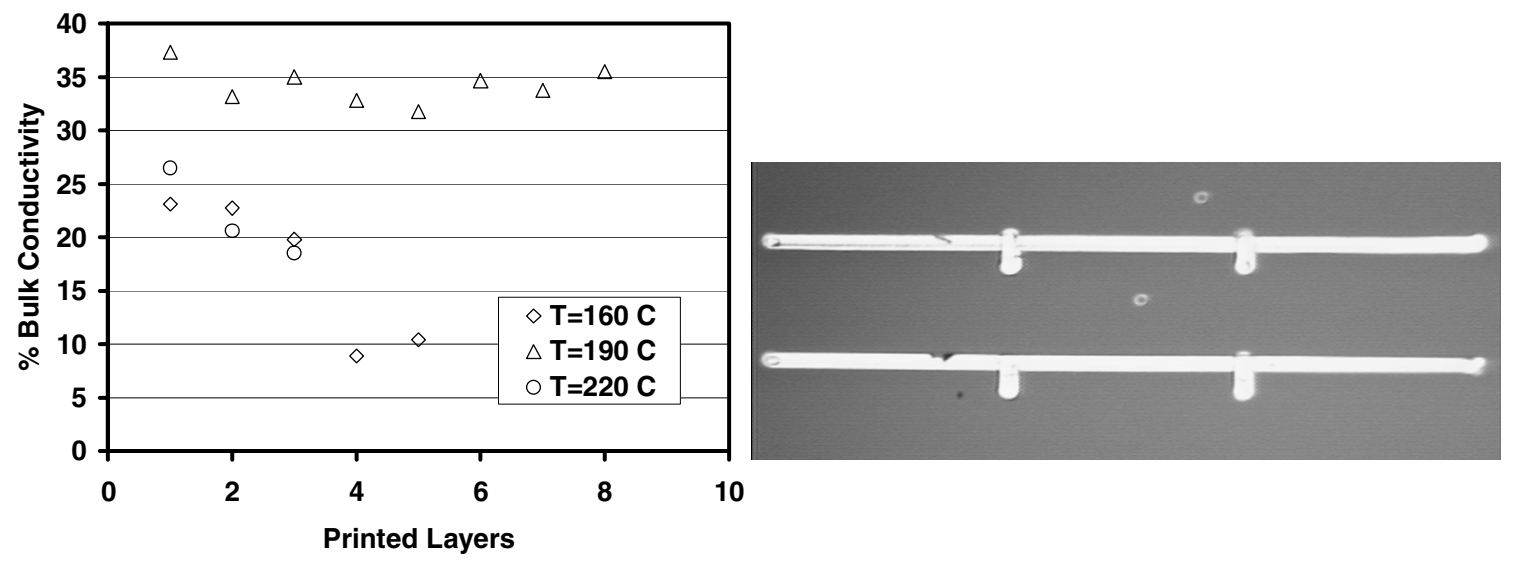

Figure 6: Variation in conductivity with temperature and number of syncopated layers (left), as measured using a four-point sheet resistance structure (right). 


\section{Dielectric Film Development}

Dielectric film development proceeded in an analogous way to the development of the conductor films above, i.e., elevated substrate temperature was used to eliminate the coffee-ring effect. However, an additional constraint is applied to dielectric films which is less critical for the conductors; in dielectrics, it is desirable to allow some reflow of the individual drops to eliminate pinholes and voids formed between drops, and also improve step coverage. Temperature therefore plays an important role in this optimization; films deposited at room temperature flow too much, and produce highly distorted films, while films deposited at temperatures that are too high produce films that have numerous pinholes, which must be subsequently filled using multiple layers. This is unacceptable for capacitors, since it results in an increase in the dielectric thickness and a consequent reduction in specific capacitance. At intermediate temperatures (the specific values of which depend greatly on the polyimide used and the concentration of the printing solution), it is possible to form films that reflow enough to fill pinholes but do not result in the formation of distorted and agglomerated films. This is shown in figure 7.
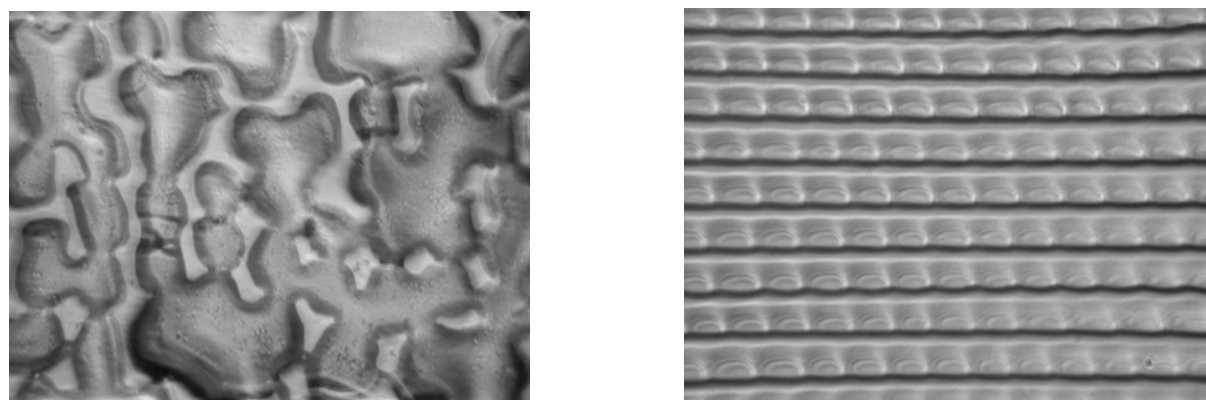

Figure 7: Printed dielectric layers showing the agglomeration that results at low temperatures (left), and the smooth films achieved at intermediate temperatures (right). At high temperatures, the lack of reflow results in pinholes.

\section{$\underline{\text { Multilaver interconnect and inductive component demonstration }}$}

To form multilayer interconnects, the conductor and dielectric technologies described above are integrated. As a representative structure, we use perpendicular crossing interconnects separated by a dielectric layer, and perform electrical measurements to determine the likelihood of shorts, indicative of pinholes. We also measure the resistance of the top conductor layer on both sides of the crossover; high resistance is indicative of poor step-coverage over the crossover by the top conductor. To ensure good step coverage by the dielectric layer, high substrate temperatures are used, with multiple passes to ensure absence of pinholes and reduced cross-talk between layers. Use of lower temperatures results in reflow off the step, increasing the likelihood of shorts between the conductor layers. As shown in figure 8, by using three layers of overlaid dielectric, it is possible to essentially eliminate shorts. Step coverage by the top conductor is excellent in all cases. Combining the dielectric and conductor technologies, we have also demonstrated a variety of inductors. Using a non-optimized layout, we have achieved Q's approaching 0.5 at $135 \mathrm{kHz}$, which is the highest value achieved using a $1 \mu \mathrm{m}$ thick conductor to our knowledge, printed or otherwise. Further results, improved optimization strategies, and integration with a robust capacitor technology will be presented shortly [5]. 

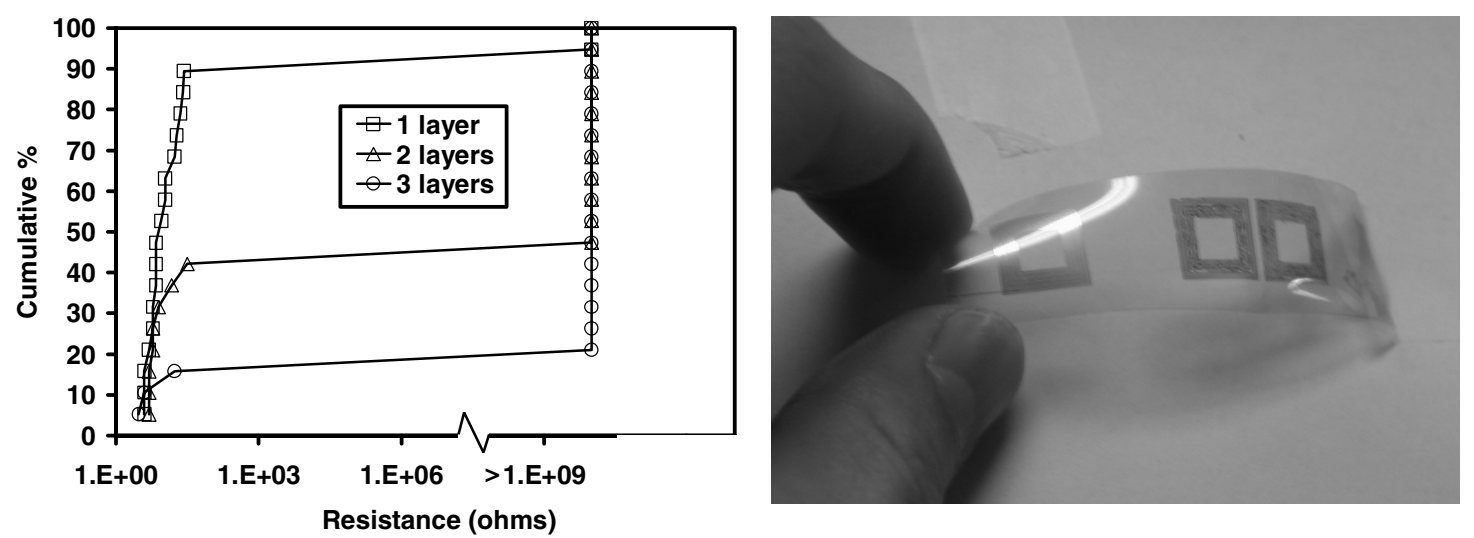

Figure 8: The conductor and dielectric technologies developed herein allow us to demonstrate multilevel interconnects with few shorts (left), and also demonstrate inductors with the highest Q's reported to date using similar geometries (right).

\section{CONCLUSIONS}

High-quality passive components are a crucial component technology required for the demonstration of low-cost all-printed RFID. Using gold nanoparticle-based conductors and polyimide dielectrics, we have developed, for the first time, a high-quality interconnect and inductive component technology that offers unprecedented levels of conductivity in a printed film, enabling the demonstration of high quality inductors and a robust multilevel interconnect scheme. By optimizing the deposition parameters, the most important of which are substrate temperature and choice of solvent, it is possible to produce low-resistance conductor lines and dielectric with excellent surface morphology and electrical characteristics. These therefore represent an important first step towards demonstration of an all-printed RFID technology.

\section{ACKNOWLEDGEMENTS}

Portions of this work were jointly sponsored in part under SRC contract 01-MC-460 and DARPA grant MD972-01-1-0021. Other parts of this work were funded by the National Science Foundation under grant ECS-0220931.

\section{REFERENCES}

[1] H. Sirringhaus et al, Science, vol. 290, pp. 2123, 2000.

[2] V. Subramanian, "Towards Printed Low-Cost RFID Tags: Device, Materials and Circuit Technologies", V. Subramanian, Proceedings of the 2nd Advanced Technology Workshop on Printing an Intelligent Future: Printed Organic and Molecular Electronic Technologies, Boston, MA, March 16-19, 2003.

[3] D. Huang, F. Liao, S. Molesa, D. Redinger, and V. Subramanian, "Plastic-compatible lowresistance printable gold nanoparticle conductors for flexible electronics", in print, J.

Electrochem. Soc.

[4] Fuller et al, J. Micromechanical Systems, vol. 11, pp. 54, 2002.

[5] D. Redinger, R. Farshchi, and V. Subramanian "Inkjetted Passive Components on Plastic Substrate for RFID”, to be presented, 2003 IEEE Device Research Conference. 